

No. 39.

Berlin, den 26. September 1907.

33. Jahrgang.

\title{
Zum XIV. Internationalen Kongreß für Hygiene und Demographie.
}

Der Internationale Kongreb für Hygiene und Demographie tagt zum ersten Male auf deutschem Boden. Vor mehr als 50 Jahren in Belgiens Hauptstadt gegründet, hat der Kongreb wiederholt den Weg zu seiner schönen Geburtsstätte angetreten, ist mehrere Male in Frankreichs herrlicher Capitale gastreich empfangen worden, hat Italien, die Schweiz, Holland, Oesterreich, Ungarn, England, Spanien aufgesucht, um nunmehr auch in unserem Vaterlande seinen vorübergehenden Wohnsitz zu nehmen. „Spät kommt Ihr - doch Ihr kom mt", und in aufrichtiger, tiefgefühlter Freude heißen wir die Mitglieder des Kongresses in den Mauern unserer Residenz herzlich willkommen.

Deutschlands Anteil an der Entwicklung der modernen Hygiene ist jünger als der ihrem Dienste sich widmende internationale Kongreb, später als in anderen Nationen ist bei uns diese kostbare Pflanze der Kultur zum Sprossen, zur Blüte gelangt. Zwar hat Johann Peter Frank schon am Ausgang des 18. Jahrhunderts sein "System einer vollständigen medizinischen Polizei“" verfaßt und sich darin als ein so vortrefflicher Mann der Wissenschaft erwiesen, daß ihm M. v. Pettenk of er gelegentlich das Lob erteilt: "Keiner vor ihm hat den Wert der Gesundheit des Einzelnen und deren Beziehung zum Gesamtwohl so scharf und so richtig angesehen und erkannt als or" -- allein sein Einflub auf den praktischen und wissenschaftlichen Ausbau der Gesundheitslehre blieb gering und überdauerte kaum die Lebensspanne seines Werkes.

Bahnbrechend in der Förderung der öffentlichen Gesundheitspflege sind während des vierten und fünften Dezenniums des 19. Jahrhunderts allen Völkern die Engländer vorangegangen. Durch die Steigerung der hygienischen und sozialen Mifstïnde in den überaus schnell sich vergrößernden Industriezentren wachgerufen, insbesondere aber infolge der starken Verheerungen, die die Cholera 1831 auch in England angerichtet hatte, warfen die englischen Aerzte sich Ende der dreiBiger Jahre mit Eifer auf das Studium der sanitären Verhältnisse in den großen Städten, man suchte die Bedingungen zu erforschen, unter denen die epidemischen Krankheiten entstehen und sich verbreiten, man machte statistische Erhebungen über Geburts- und Todesfälle, man beschäftigte sich mit den Lebensverhältnissen der Arbeiterbevölkerung, die in den Werkstätten der zu reicher Blüte sich entfaltenden In- dustrie an Zahl außerordentlich zugenommen hatte und fortdauernd im Wachstum begriffen war. Die Medizinalstatistik wurde von einem Registrar General of Births, Deaths and Marriages (1836) verwertet, ein vom Parlament eingesetztes Commitee on the Health of Towns leitete die Reorganisation der städtischen Gesundheitspflege. Es wurde ein Zentralgesundheitsamt (General Board of Health) errichtet, dessen Mitglieder - die Superintendent Inspectors - mit exekutiver Machtbefugnis ausgestattet wurden, und für die einzelnen Bezirke wurden die Locals Boards of Health - Ortsgesundheitsämter - geschaffen, deren Beamte, die Officers of Health, in ihren kleinen Wirkungskreisen die sanitären Verhältnisse zu überwachen hatten.

Auf Grund aller bei diesen Studien gewonnenen Ergebnisse wurde ein Staatsgesetz (Public Health 1848) und kommunale Verordnungen erlassen, mit Hilfe deren hygienische Reformen im großen Stil durchgeführt wurden. Die Wasserver. sorgung wurde zentralisiert und dabei den Anforderungen an ein gutes Trink- und Nutzwasser nach Möglichkeit angepaßit, die Abfuhr der Fäkalien und sonstigen Abfallstoffe wurde durch unterirdische Kanäle geleitet, man legte ungesunde Wohnhäuser und Straßen nieder und ersetzte sie durch zweckmäßigere Bauten. Die Krankenhäuser, deren Anlage schon im 18. Jahrhundert zuerst in England durch die Umwandlung des Korridor- in das Pavillonsystem erheblich gebessert war, wurden weiter vervollkommnet.

Die Erfolge dieser grobartigen Reformen, die sich namentlich durch ein Absinken der Morbiditäts- und Mortalitätsziffer in zahlreichen Landesteilen manifestierten, blieben naturgemäß nicht ohne Einflub auf die übrigen Nationen. Auch bei ihnen verstärkte die Not, welche die opferreichen Seuchen erzeugten, die Nacheiferung. Am deutlichsten offenbarten sich diese Bestrebungen in dem Zusammenschlub der Nationen zu gemeinsamem Kampfe gegen die aus dem Orient immer wieder nach Europa einbrechenden Epidernien. So wurde schon 1840 der Internationale Gesundheitsdienst im türkischen Reich unter der Aufsicht des „Obersten Gesundheitsrats“ eingerichtet, so wurde - auf die sehr verdienstvolle Initiative Frankreichs - im Jahre 1851 die erste internationale Sanitatskonvention zur Abwehr der Pest, der Cholera und des Gelb. fiebers abgeschlossen. 
Auch in Deutschland blieb das ruhmvolle Vorgehen der "Vettern jenseits des Kanals“ nicht unbeachtet, und der Begriff der "öffentlichen Gesundheitspflege $e^{*}$ fing an für die Aerzte einen gröferen Inhalt zu gewinnen. Am schärfsten und frühesten wurde die hohe Bedeutung der englischen Reformen von dem universellen Genie Rudolph Virchows erfabt und zum Ausdruck gebracht. In seiner 1848 (mit R. Leubuscher) gegründeten Wochenschrift „Die medizinische Reform" trat der 27jährige Forscher mit der ihm eigenen feurigen Energie und zwingenden Beweiskraft immer wieder für eine Umwandlung unserer sanitären Verhältnisse ein. Hatte er doch die praktische Tragweite der Hygiene bei seinem zu Anfang desselben Jahres im Auftrage des Unterrichtsministers unternommenen Studium des oberschlesischen Hungertyphus genügend kennen gelernt! Aus seinen vier Artikeln, betitelt "Die öffentliche Gesundheitspflege", (No. $5 \mathrm{ff}$. seiner Wochenschrift) sei es gestattet hier kurz folgende interessante Bemerkungen wörtlich wiederzugeben:

Es genügt nicht, daß der Staat jedem Staatsbürger die Mittel zur Existenz. überhaupt gewährt, daB er daher jedem, dessen Arbeitskraft nicht ausreicht, sich diese Mittel zu erwerben, beisteht; der Staat muB mehr than, er muß jedem so weit beistehen, daß er eine gesundheitsgemäße Existenz habe. Das folgt einfach aus dem Begriff des Staats als der sittlichen Einheit aller Einzelnen, aus der solidarischen Verpflichtnng Aller für Alle."

${ }_{n}$ Epidemien gleichen großen Warnungstafeln, an denen der Staatsmann von großem Styl lesen kann, daß in dem Entwicklungsgange seines Volkes eine Störung eingetreten ist, welche selbst eine sorglose Politik nicht länger übersehen darf.“

„Für die bedürftigen Arbeitsunfähigen also, wie der französische Verfassungsentwurf sagt, für verlassene Kinder, Sieche und Greise muß der Staat unzweifelhaft sorgen. Der öffentlichen Gesundheitspflege fällt hier zunächst die Entscheidung zu, ob man dieser Sorge in eigenen Anstalten (Gebär-, Findel-, Waisen-, Kranken-, Siechen-, Invalidenhäusern), oder in einer đem Familienleben sich anschließenden Weise nachkommen soll; sodann wie weit in jedem dieser Fälle die Verpflichtungen des Staates gehen und durch wen sie vertreten sein sollen, in welcher Art sie auszuführen sind etc."

${ }_{n}$ Die öffentliche Gesundheitspflege hat gegenüber den Arbeitern noch ganz andere und nähere Aufgaben. Schon die Breslauer Denkschrift, sowie Hr. Leubuscher (No. 3) hat es angedeutet, daß ein Gesetz über die Arbeitszeit der verschiedenen Altersklassen von der Medizin ausgehen muB; sie hat deutlich gezeigt, in welcher Vernachlässigung sich unsere Gesetzgebung über nachtheilige Gewerbe befindet. In England stirbt durchschnittlich jährlich einer aus 45 Menschen, in den Fabrikdistrikten (Cheshire, Lancashire, Yorkshire) 1 aus 39,8, in Edinburg 1 aus 29. In Liverpool war 1840 die durchschnittliche Lebensdaner der höheren Klassen 35, die der Geschäftsleute und besser gestellten Handwerker 22, die der arbeitenden und dienenden Klassen I5 Jahre. Und doch, trotzdem daß zwei Drittheile der englischen Bevölkerung durch die Industrie in Anspruch genommen werden, hat England nach der Zählung von 1843 nur eine Mortalität von 21,85 p. Mille, während Frankreich 23,61 und Preußen 27,09 p. Mille aufwies !“

${ }_{n}$ Haben wir also nicht hier würdige Aufgaben für die öffentiche Gesundheitspflege? Gewährt nicht die jetzige Lebensweise unseres Volkes die vielfachsten Anknüpfungspunkte für große Verbesserungen? Ist nicht in Kleidung, Nahrung und Wohnung unendlich viel zu reformieren?

Wir selbst haben uns bemüht, an dem Beispiel von Oberschlesien die Notwendigkeit gründlicher Veränderungen in der $\mathrm{Hy}$ giene der ländlichen -Wohnungen, in der Regulirung des Stromlaufs, der Entwässerung von Sümpfen und Wiesen etc. zu zeigen. So verheerende Krankheiten, wie Typhus, Tuberkulose und Wechselfieber, müssen aufhören, in einer solchen Ausdehnung, wie bisher, unter unserem Volke zu herrschen.

Sollen wir endlich noch an die Beziehung der öffentlichen Gesundheitspflege zu dem öffentlichen Unterricht erinnern? Nicht bloß die physische Erziehung, die Gymnastik in ihrer weitesten Ausdehnung, die Bestimmung der Unterrichtszeit gehören hierher, sondern der Unterricht selbst muß gewisse Impulse von der $\mathrm{Me}$ dizin erhalten. Populäre Unterweisungen, die eine allgemeine, vernünftige Diătetik, eine allgemeinere Prophylaxe etc. begründen, müssen sich auf eine durch den Unterricht allgemeiner verbreitete Kenntniss des menschlichen Körpers und seiner Verrichtungen stlitzen; die Sittlichkeit muß aus einer gründlicheren Anschauung von dem Wesen der Natureischeinungen, von der Bedeutung der ewigen Naturgesetze und von ihrer Geltung im eigenen Leibe neue und sicherere Stiutzen gewinnen.
Das ist ein kurzer und nicht einmal ganz umfassender Ueber. blick von dem Umfange der öfentlichen Gesundheitspflege."

In der Tat - kein "ganz umfassender Ueberblick“, aber ein Programm, aus welchem hervorgeht, wie klar der junge Gelehrte die wesentlichen Aufgaben einer wirksamen Hygiene erfaßt hat, wie deutlich die Forderungen einer notwendigen Reform der öffentlichen Gesundheitspflege in seinem Geiste präzisiert waren. Er war gewiß der Mann, der es verınocht hätte, diese Reform in grofartigem Maßstabe durchzuführen und die Hygiene auf eine hohe Stufe zu erheben: allein sein Genius fesselte ihn bei der pathologischen Anatomie und der allgemeinen Pathologie, auf deren Gebiete or 1848 bereits Arbeiten von größter Bedeutung geschaffen hatte. Welche unvergänglichen Verdienste er sich trotzdem um die Entwicklung der öffentlichen Gesundheitspflege erworben hat, ist bekannt, auch bei Gelegenheit seines 80 . Geburtstages in dieser Wochenschrift genauer dargelegt worden. Wenn die auswältigen Mitglieder des XIV. Internationalen Hygiene-Kongresses sich bei ihrer Anwesenheit in den Mauern „der reinlichsten aller Hauptstädte" an vielen mustergültigen sanitären Einrichtungen erfreuen werden, so werden sie wissen, dab sie zumeist erstanden sind unter der Initiative und Leitung des "Stadtverordneten" Rudolph Virchow und noch erstehen auf der Basis seiner grundsätzlichen Anschauungen: sei es die Kanalisation, sein gröbtes kommunales Werk, das - ill den siebziger Jahren begonnen - die ungeteilte Bewunderung aller Hygienikel fand und findet, oder die Wasserleitung, oder die Krankenhäuser, von denen das Krankenhaus am Friedrichs. hain - seinerzeit eines der schönsten und mustergültigsten Europas - unter seiner Mitwirkung erbaut wurde, oder die Gemeindeschulen etc. etc.

Die Bedeutung, welche Virchow für unsere moderne $\mathrm{P} \lambda$ thologie besitzt, hat für die öffentliche Gesundheits. pflege Max v. Pettenkofer gewonnen: er ist unbestritten der Begründer der wissenschaftlichen Hygiene. Die sanitären Fortschritte in England und anderen Kulturnationen waren, so hoch sie auch bewertet werden müssen, doch nur auf einer empirischen Basis errungen, sie waren auf unbewiesene Hypothesen aufgebaut; die späteren experimentellen Prüfungen ergaben Resultate, die mit den praktischen Erfahrungen oft nicht übereinstimmten, und die tatsächlichen Beobachtungen selbst wiesen nach Ort und Zeit erhebliche Abweichungen und Widersprüche auf: ganz besonders bei der Pathogenese und Bekämpfung der Infektionskrankheiten. Hier schuf die von v. Pettenk of or begründete Reform Wandel. Mit Hilfe der zu seiner Zeit schon reicher entwickelten Methoden der Naturwissenschaften, der Chemie, Physik, Botanik und nicht zum mindesten der Physiologie - deren bedeutender Vertreter Carlv. Voit neben und mit v. Pettenk ofer an der Münchener Universität wirkte - führte v. Pettenkofer das exakte wissenschaftliche Experiment in die hygienischen Untersuchungen ein, er suchte auf diese Weise die Wirkungen des Wassers, der Wohnung, der Nahrung, der Kleidung etc. auf den menschlichen Organismus und seine Gesundheit zu erforschen, er bemühte sich experimentell die Beziehungen del Luft, der Nahrungsmittel und des Bodens zu den Infektionskrankheiten, insbesondere zu der 1854 in Bayern herrschenden Cholera und zum Typhus zu ergründen, und so erhob er die Hygiene zu einem wichtigen Gliede der Naturwissenschaften selbst. Seine Anschauungen über die Hygiene und ihre Aufgaben hat $v$. Pettenkofer in seinem Aufsatze "Ueber Hygiene und ihre Stellung an den Hochschulen“ (Wiener medizinische Wochenschrift 1875 , No. 6 ff.) in lichtvoller Klarheit dargelegt.

Ich fasse die Hygiene als Wirtschaftslehre von derGesundheit auf, ganz ähnlich, wie die Nationalökonomie die Güter. wirtschaft betrachtet. Wie in der Nationalökonomie nicht bloß die Furcht vor der Einbuße, sondern noch vielmehr das Streben nach höherem Gewinn die treibende Kraft ist, so muß es auch in der Hygiene als Gesundheitslehre werden. Die Hygiene hat die Wertig. keit aller Einflise der natïrlichen und kunstlichen Umgebung des Organismus zu untersuchen und festzustellen, um durch diese $\mathrm{Er}$. kenntniß dessen Wohl zu fördern."

Gleichwie im Laufe der Zeit aus den Kameralfachern eine Wirtschaftslehre entstanden ist, so muß aus der Gesundheitspflege und Medizinalpolizei eine Gesundheitslehre sich entwickeln ... 
Es wird auch die Zeit kommen, in welcher der Hygieniker mit seiner Theorie und Praxis ebenso ebenbürtig in den medizinischen Fakultäten stehen wird, wie die Vertreter anderer, alterer Fiacher der Medizin. Diese Zeit kann aber nur kommen, wenn die medizinischen Fakultäten allmählich der Pflege der Hygiene die rleiche Sorgfalt angedeihen lassen, wie diesen Fächern."

Das in den letzterwähnten Worten bezeichnete Ziel zu erreichen, ist v. Pettenk of er selbst schon dank seiner genialen Schaffenskraft gelungen, durch inn ist der hygienische Unterricht organisiert und als gleichwertiger Lehrgegenstand in die Universitäten eingeführt worden. Auf Grund einer von ihm 1862 ausgearbeiteten Denkschrift faßte die medizinische $\mathrm{Fa}$ kultät Münchens die folgenden Beschlüsse:

1. Es sind die Vorträge über Medizinalpolizei durch Vorträge über Hygiene zu ersetzen. 2. Hygiene bildet ein Nominalfach, dessen Vertretung nicht notwendig wie bisher dem Vertreter der gerichtlichen Medizin als Professor der Staatsarzneikunde anheimfallt 3. Hygiene ist als Gegenstand des Fakultätsexamens aufzunehmen.

1865 wurde v. Pettenk ofer der erste Ordinarius für Hygiene in Deutschland, nach ihm erhielten in demselben Jahre J. Scherer in Würzburg, Gor up-Besanez in Erlangen das Ordinariat. Längere Zeit dauerte es noch, bis v. Petten k of er eine der Bedeutung seines Schaffens würdige Arbeits stätte erhielt: erst im Jahre 1878 konnte das erste deutsche hygienische Institut in München eröffnet werden. Ihm reihten sich in bald schneller, bald langsamer Folge die Institute auf den anderen deutschen Universitäten an. Der Begründung der einzelnen Institute in den Bundesstaaten ging die Errichtung des Kaiserlichen Gesundheitsamts in Berlin (1876) vorauf, dem nach der an den Bundesrat gerichteten Denkschrift des Reichskanzlers (vom 9. April 1872) die Aufgabe zufallen sollte:

das Reich in der Ausúbung der ihm zugewiesenen Aufsicht iiber die medizinal- und veterinăr-polizeilichen Angelegenheiten zu unterstützen, von den hierfür in den einzelnen Bundesstaaten bestehenden Einrichtungen Kenntnis zu nehmen, die vom Reiche ausgehende Gesetzgebung vorzubereiten, die Wirkungen der im Interesse der öffentlichen Gesundheitspflege ergriffenen Maßnahmen zu beobachten, in geeigneten Fallen den Staats- und Gemeindebehörden Auskunft zu erteilen, die Entwicklung der Medizinalgesetzgebung in außerdeutschen Ländern zu verfolgen, die Herstellung einer genügenden medizinischen Statistik für Deutschland zu organisieren.

In seiner oben erwähnten Abhandlung "Ueber Hygiene und ihre Stellung an den Hochschulen" sagte Max v. Pettenk ofer: „Einstweilen haben wir es als einen faktischen Zustand zu betrachten, und mit diesem Faktor zu rechnen, daß es Hauptaufgabe der Hygiene sei, Krankheiten zu verhüten. Um dies zu vermögen, muß man die Ursachen und Veranlassungen zu Krankheiten kennen und sie beseitigen lernen. Aetiologie und Prophylaxis müssen daher die Hygiene zunächst interessieren." So bedeutungsvoll aber die Untersuchungen waren, die Pettenk ofer auf die Erforschung dieser von ihm als wichtigste bezeichneten Aufgabe verwandte sie zu lösen war ihm, dem hervorragenden Meister der $\mathrm{Hy}$ giene nicht vergönnt, dieses unsterbliche Verdienst sich zu erwerben war beschieden dem genialen Begründerder modernen Bakteriologie, Robert Koch. Seine klassischen Studien haben uns die Erreger verderblichster Volksseuchen kennen gelehrt, durch ihn sind uns die Methoden einer rationellen, wissenschaftlich begründeten Desinfektion und Seuchenbekämpfung in die Hände gegeben, von ihm und seinen Schülern sind wir befähigt worden, unseren Organismus gegen die Infektion mit Krankheitserregern zu schützen und ausgebrochene Infektionskrankheiten zu heilen. Von der Veröffentlichung des Wollsteiner Kreisphysikus „Ueber die A etiologie der Wundinfektionskrankheiten" im Jahre 1878 datiort die jüngste, für die Erkennung und Bekämpfung der menschlichen Infektionskrankheiten wichtigste Epoche der Hygiene. Welche ungeheuren Fortschritte in ihren drei Dezennien erzielt worden sind, das möge die kurze Erwägung beleuchten: welche Panik die ganze Kulturwelt noch vor wenig mehr als einem halben Jahrhundert ergriff, wenn die Cholera im fernsten Osten ihr Haupt erhob, und mit welcher sicheren und ungestörten Ruhe wir heute während der Tagung des XIV. Internationalen Hygiene-Kongresses in Berlin die Botschaft vernehmen, dab die
Seuche, wenige hundert Meilen entfernt, nahe der Grenze Preußens ihre unheimliche Tätigkeit entfaltet hat!

Die einzelnen Grobtaten Robert $\mathrm{Kochs}$ in dieser kurzen historischen Skizze auch nur flüchtig zu schildern, können wir uns versagen, nicht nur weil sie gelegentlich der Feier seines sechzigsten Geburtstages vor vier Jahren in einem Aufsatz L oefflers hier gewürdigt worden sind, sondern auch weil die 32 Jahrgänge unserer Wochenschrift selbst eine lehrreiche Chronik seines Wirkens und der gesamten letzten Periode der Hygiene darstellen. Von ihrer Begründung im Jahre $1875 \mathrm{ab}$ hat diese Zeitschrift der öffentlichen Gesundheitspflege ihr Hauptaugenmerk zugewandt, getreu dem Ziele, das ihr Paul Börner, ihr geistvoller und tatkräftiger Begründer, gesteckt und für dessen Verfolgung er mit seinen besten Krafften während seiner ganzen erfolgreichen Wirksamkeit eingetreten ist. Am Tage der Eröffnung der hochbedeutsamen „Allgemeinen Deutschen Ausstellung a d dem Gebiete der Hygiene und des Rettungswesens", an deren Schöpfung Paul Börner so hervorragenden Anteil hatte - eine eigentümliche Fügung des Schicksals läfit den XIV. Internationalen Hygienekongreß gerade 25 Jahre später auf demselben Boden wie jene tagen -, sehrieb er:

"Die Medizin im engeren Sinne gerät in Gefahr, zu erstarren, dogmatisch zu werden, wenn sie nicht fortdauernd im Kontakte mit den Naturwissenschaften und mit der Gesundheitspflege bleibt. Was erstere anlangt, so ist es die Aufgabe der speziell medizinischen Kongresse, die Verbindung mit der Naturwissenschaft immer von neuem und immer inniger zu knüpfen, und diese Vereine haben bis jetzt sich dieser Aufgabe wohl gewachsen gezeigt. DaB auch die Verbindung der Medizin mit der öffentlichen Gesundheitspflege, welche ebenso wichtig ist, in gleicher Weise sich immer enger und fester knüpfe, dazu wird die Hygiene-Ausstellung einen mächtigen und hoffentlich dauernden Antrieb geben."

Der grobe Erfolg, den die Deutsche Hygiene-Ausstellung des Jahres 1882/83 für die Entwicklung der öffentlichen Gesundheitspflege nicht nur in Deutschland, sondern auch in den übrigen Kulturstaaten gezeitigt hat, hat Börners Voraussage in Erfüllung gehen lassen: heute, in den Tagen des XIV. Internationalen Hygiene-Kongresses ist das Band zwischen Hygiene und Medizin unlöslich geworden. Jeder Arzt fühlt sich heute als ein Anwalt und Förderer der Gesundheitspflege, sieht seine Aufgabe darin, die Krankheiten nicht .nur zu heilen, sondern sie auch nach besten Kräften zu verhüten. Die Staatsregierungen der Kulturvölker sind sich ihrer Verantwortung in allen Fragen des öffentlichen Sanitätswesens voll bewubt, sie sind von der Ueberzeugung durchdrungen, daf die Hygiene einen integrierenden Bestandteil der Nationalökonomie bildet, daß die Gesundheit des Bürgers das wichtigste Kapitalvermögen des Staates darstellt. Von diesem Gesichtspunkte aus wird die sanitäre Gesetzgebung, der Unterhalt der öffentlichen hygienischen Institutionen, der hygienische Unterricht an den Hochschulen mit Ernst und Sorgfalt ständig gepflegt. Von demselben Bestreben hat Deutschland sich auch bei der Inauguration seiner grobartigen sozialen Gesetzgebung (1884) leiten lassen, die vorbildlich für alle anderen Nationen geworden ist.

Das Studium des Problems: unserer irdischen Gïter köstlichstes, die Gesundheit, zu schützen und unsere physischen Lebensbedingungen immer zweckmäfiger zu gestalten, bildet auch das Leitmotiv für die Verhandlungen des XIV. Internationalen Kongresses für Hygiene und Demographie. Aus allen Ländern des Erdballs sind Tausende von Männern der Wissenschaft und Praxis hierher zusammengeströmt, um gemeinsam im Dienste der Kultur zu arbeiten. Dank der auggezeichneten Vorbereitungen des Organisationskomitees, unter der Leitung des Präsidenten des Kaiserlichen Gesundheitsamts Dr. Bu m m, dank der gesicherten Anteilnahme der hervorragendsten in- und ausländischen Forscher wird der Verlauf des Kongresses - das kann man ohne Uebertreibung vorhersagen - den hochgespannten Erwartungen entsprechen, und seine wissenschaftlichen Ergebnisse werden einen Fortschritt in vielen interessanten und wichtigen Fragen bilden. Möge auch dieser internationale KongreB ein lorbeerbekränzter Markstein werden auf dem unendlichen Wege der Menschheit zu ihren höchsten Idealen. J. Schwalbe. 